\title{
Activation and repression of mammalian gene expression by the c-myc protein
}

\author{
Rima Kaddurah-Daouk, ${ }^{1}$ John M. Greene, ${ }^{1}$ Albert S. Baldwin, Jr., ${ }^{2}$ and Robert E. Kingston ${ }^{1}$ \\ 'Department of Genetics, Harvard Medical School and Department of Molecular Biology, Massachusetts General Hospital, \\ Boston, Massachusetts $02114 ;{ }^{2}$ Center for Cancer Research and Department of Biology, Massachusetts Institute of \\ Technology, Cambridge, Massachusetts 02139
}

\begin{abstract}
One mechanism by which nuclear-localized oncogenes might transform cells is through an ability to regulate gene expression. We show that the c-myc protein stimulates the level of appropriately initiated expression from the human heat shock protein 70 (hsp70) promoter. Sequences required for full activation lie upstream of the transcription initiation site and are distinct from sequences necessary for basal expression. These sequences also appear distinct from promoter sequences necessary for heat induction, serum induction, and induction by the papovavirus $\mathrm{T}$ antigens. The c-myc protein inhibits appropriately initiated expression from the mouse metallothionein I (MT-I) promoter. A mutation that removes 138 amino acids of exon 2 produces a c-myc gene product that is capable of activating the hsp 70 promoter but is no longer capable of inhibiting MT-I expression, suggesting that these two properties reside in different domains of the c-myc protein. Expression from the adenovirus EII promoter is slightly inhibited, while expression from the SV40 early promoter is minimally affected by the c-myc protein. Both the spectrum of promoters regulated by the c-myc protein and the sequence requirements for that regulation differ from those of previously characterized viral trans-activating proteins. The data suggest that the c-myc protein can both stimulate and inhibit transcription from mammalian promoters in a novel manner.
\end{abstract}

[Key Words: c-myc protein; hsp70 promoter; oncogenes; transcription regulation; metallothionein I promoter]

Received January 30, 1987; revised version received and accepted March 31, 1987.

Many viral and cellular oncogenes encode protein products that have been found to reside in the nucleus. Several viral representatives of this class have been shown to regulate gene expression (for review, see Kingston et al. 1985). The most extensively characterized of these, the adenovirus Ela gene products, have been shown to modulate promoter activity both positively and negatively (Berk et al. 1979; Jones and Shenk 1979; Borelli et al. 1984; Velcich and Ziff 1985). These observations have led to the proposal that nuclear-localized oncogene products exert their transforming phenotype by modulating expression of a set of genes important for growth control. One prediction of this hypothesis is that cellular oncogenes will share with viral oncogenes an ability to regulate gene expression.

Several lines of evidence suggest that the cellular oncogene $c-m y c$ may play such a regulatory role. The protein is localized to the nucleus and appears to be a DNA-binding protein, although no specificity for this binding has yet been shown (Abrams et al. 1982; Donner et al. 1982; Alitalo et al. 1983; Hann et al. 1983; Persson and Leder 1984). This protein shares with the trans-activating regions of the DNA tumor viruses the ability to complement c-Ha-ras in transforming primary cells (Land et al. 1983; Ruley 1983). Most notably, the product of a rearranged mouse c-myc gene stimulates expression of a chimeric gene containing a Drosophila heat shock protein 70 (hsp70) promoter region (Kingston et al. 1984a). While it was not possible to determine whether this stimulation was at the level of appropriately initiated transcription, this observation raised the possibility that the c-myc protein might stimulate transcription from mammalian hsp 70 promoters.

The human genome encodes several hsp70 loci (for review, see Pelham 1986). The particular promoter used here (Wu et al. 1985) is stimulated when cells respond to heat shock as well as when resting cells are fed fresh serum (Wu and Morimoto 1985; Wu et al. 1986a). This promoter is also stimulated by the products of the adenovirus EIa gene and the polyomavirus and SV40 early regions, arguing that it is a reasonable candidate for stimulation by a cellular oncogene (Nevins 1982; Kao and Nevins 1983; Imperiale et al. 1984; Khandjian and Turler 1983; Kingston et al. 1986; Wu et al. 1986b). The sequences responsible for serum stimulation lie within 60 bases of the transcription start site, whereas those responsible for heat induction are centered at -100 (Wu et al. 1986a). The sequences necessary for stimulation of this hsp70 promoter by the polyomavirus early region lie within -70 , and are not separable from those required for basal expression of the promoter (Kingston et al. 1986).

We demonstrate here that the c-myc gene product stimulates appropriately initiated expression from this 
human hsp70 promoter. In contrast with activation by the papovavirus $T$ antigens, sequences required for maximal induction of the promoter by the c-myc protein are distinct from those required for basal activity. We also show that the level of appropriately initiated expression from the mouse metallothionein I (MT-I) promoter is inhibited by the c-myc protein, implying that this protein can both positively and negatively regulate gene expres. sion.

\section{Results}

The $c$-myc protein stimulates expression from the human hsp70 promoter

To determine the regulatory capabilities of the c-myc protein, we performed transient cotransfection experiments. We included three plasmids in each experiment. All transfections contain a plasmid ( $\mathrm{p} \Delta$ series) in which the human hsp70 promoter is fused to the reporter gene chloramphenicol acetyltransferase (CAT; Fig. 1). A second plasmid differs for each transfection, and either expresses the c-myc gene product (pSV2-cmyc, Land et al. 1983), produces a c-myc message that contains a frameshift mutation at amino acid 40 (pfsmyc-20), or contains only vector DNA (pUC13). We measure the effect of the c-myc protein on hsp70 expression by comparing the level of CAT activity in the presence and absence of cotransfection with pSV2-cmyc. The use of plasmid pfsmyc-20 as a control ensures that we are measuring a characteristic of the c-myc protein.

We wished to compare the effects of the c-myc protein on expression of various promoters. Initial results suggested that transcription from the mouse MT-I promoter was repressed by the c-myc protein. To compare directly the effects of the c-myc protein on hsp70 and MT-I transcription, a third plasmid, pXGH5, was included in each experiment (Selden et al. 1986). This plasmid contains a chimeric gene in which the human growth hormone (hGH) coding region is fused to the mouse MT-I promoter (Durnam et al. 1980; Denoto et al. 1981; Hamer and Walling 1982). The human growth hormone (hGH) gene product is secreted, and the amount produced can be easily quantitated by a radioimmune sandwich assay. The amount of CAT made from the human hsp70 promoter and the amount of hGH made from the mouse MT-I promoter can thus be measured in the same transfection. We can therefore calculate a ratio between hsp70 expression and MT-I expression in each transfected dish, and determine how the c-myc protein affects this ratio.

The results of a typical experiment are shown in Table 1. The transfections in which pSV2-cmyc (set 2) or pfsmyc-20 (set 3 ) is the cotransfected plasmid are identical except for the four-base deletion in pfsmyc-20 that precludes synthesis of the complete c-myc gene product. Comparison of these two transfections demonstrates that expression of the complete c-myc protein increases CAT expression 3.3-fold. Note that cotransfection with pfsmyc- 20 results in a two- to threefold stimulation of CAT activity relative to that seen after cotransfection with pUC13 (see also Fig. 4). This effect is highly reproducible. It might be due to an activity of the 40-aminoacid fragment of c-myc expressed from this vector. Alternatively, the SV40 enhancer region contained on the expression vector may stimulate expression of the hsp70 promoter after recombination between the transfected

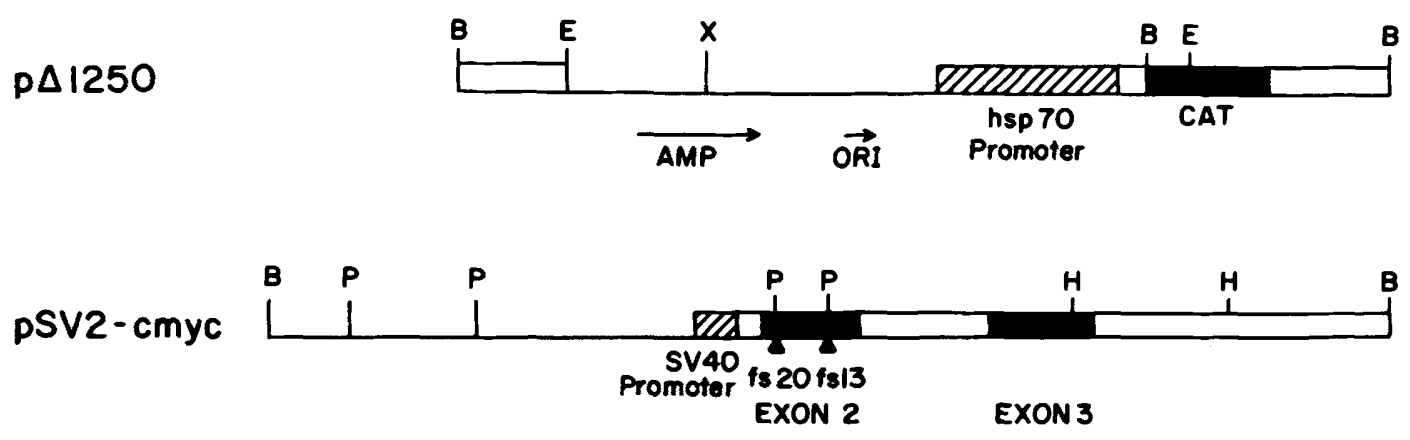

PXGH5

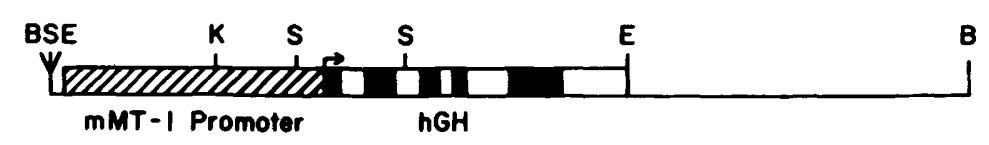

$\mathrm{I} \mathrm{Kb}$

Figure 1. Plasmids used in transfection experiments. Hatched areas show promoter regions, open boxes denote noncoding regions, filled boxes denote coding regions, and lines denote vector sequences. Plasmid p $\Delta 1250$ contains 1250 bp of $5^{\prime}$-flanking sequence of the human hsp70 promoter fused to the bacterial CAT gene at +160 . Plasmid pSV2-cmyc contains the SV40 early promoter fused to the second and third exons of a c-myc gene isolated from a mouse plasmacytoma (Shen-Ong et al. 1982; Land et al. 1983). Frameshift mutants fsmyc-20 and fsmyc-13 were generated at the indicated PstI sites by introducing 4-bp deletions (Kingston et al. 1984). pXGH5 expresses the hGH coding region fused to a mouse MT-I promoter (Selden et al. 1986). (B) BamHI; (E) EcoRI; (H) HindIII; (K) KpnI; (P) PstI; (S) SacI; (X) XmnI. 
Table 1. Modulation of human hsp70 and mouse MT-I promoter expression by the c-myc protein

\begin{tabular}{lccc}
\hline & $\begin{array}{c}\text { (Set 1) } \\
\text { pUC13 }\end{array}$ & $\begin{array}{c}\text { (Set 2) } \\
\text { pSV2-cmyc }\end{array}$ & $\begin{array}{c}\text { (Set 3) } \\
\text { pfsmyc-20 }\end{array}$ \\
\hline CAT activity & 9 & 69 & 21 \\
hGH level & 48 & 17 & 62 \\
CAT/hGH & 0.2 & 4.0 & 0.3 \\
\hline
\end{tabular}

Plasmid $\mathrm{p} \Delta 1250(8 \mu \mathrm{g})$ and $\mathrm{pXGH5}(2 \mu \mathrm{g})$ were transfected with $5 \mu \mathrm{g}$ of either pUC13 (set 1), pSV2-cmyc (set 2), or pfsmyc-20 (set 3 ) into BALB/c 3T3 cells. Forty-eight hours later, the level of $\mathrm{hGH}$ secreted into the media was determined by radioimmunoassay and CAT activity was assayed in cell lysates. Each number is the average of triplicate plates. CAT values are percent conversion; hGH values are ${ }^{125}$ I counts $\times 10^{-3}$. Values outside the linear range were determined by correction after serial dilution to within the linear range. Note that plasmid pfsmyc-20 has approximately a twofold stimulatory effect on both hsp70-CAT and MT-I-hGH expression. This effect is reproducible and is seen with every tested promoter.

DNAs. The latter possibility is supported by the observation that cotransfection with pSV2-gpt has a similar effect (data not shown). Thus, the approximately eightfold stimulation observed in this experiment when expression of the hsp70 promoter is cotransfected with pSV2-cmyc as opposed to pUC13 probably does not reflect solely an effect of the c-myc protein, but contains a component due to the expression vector used. To insure that the effects we are studying are due to expression of the c-myc protein, it is therefore critical to compare transfections in which pfsmyc-20 is transfected as a control plasmid.

Expression from the MT-I-hGH gene decreased 3.6fold after cotransfection with pSV2-cmyc. The ratio of CAT activity to hGH activity thus changes 12 -fold when these two transfections are compared. As both transfections contain the same amount of the hsp70-CAT gene and the MT-I-hGH gene, these data imply that the c-myc protein causes the hsp70 promoter to be utilized preferentially over the MT-I promoter.

Variability of the stimulatory effects of the c-myc gene product

When experiments of the sort presented in Table 1 were repeated numerous times over a 6-month period, we consistently found that the c-myc protein preferentially stimulated hsp70 promoter-driven CAT expression over MT-I promoter-driven hGH expression (Fig. 2C). There was variability in the degree of the effect, however. The degree of stimulation of CAT activity (Fig. 2A) varied between twofold and 32-fold (average sixfold), the inhibition of hGH activity (Fig. 2B) varied between 1.3-fold and 12 -fold (average 3.6 -fold), and the ratio CAT : hGH, reflecting the preferential utilization of the human hsp70 promoter, varied between threefold and 72-fold (average 20 -fold). Note that in every experiment done, this ratio was greater than 1 . The preferential stimulatory effect of the c-myc gene product was thus highly reproducible - only the degree of the effect was not.
One potential explanation for this variability is that when transfection efficiency is high, the introduction of $8 \mu \mathrm{g}$ of hsp 70 containing DNA produces near-maximal transcription from this promoter. Very little stimulation by c-myc could then be observed. To test this hypothesis, we transfected cells with increasing amounts of the human hsp70 CAT construct while holding the amount of either pSV2-cmyc or pfsmyc-20 constant. The degree of inhibition of hGH expression from the constant amount of pXGH5 in each transfection did not change in this experiment (data not shown). In contrast, the degree of stimulation of CAT expression decreased with increasing promoter concentration, as did the internal ratio CAT : hGH (Fig. 3). When several transfections were done using 1 or $2 \mu \mathrm{g}$ of $\mathrm{p} \Delta 1250$, the degree of variability was reduced, but significant variation still occurred (data not shown).

Does c-myc activate via the heat shock pathway?

Protocols using varying amounts of the hsp 70 promoter, such as described above, were used to determine the sequence requirements for stimulation of the hsp70 promoter by the c-myc gene product. We focused on determining the role of sequences upstream of those required for basal expression of the hsp 70 promoter. In particular, we wished to address the possibility that c-myc induced the hsp70 promoter by stressing the cell and thus inducing the heat shock response. We were concerned that any difference we saw between activation of two constructions would reflect the variability in activation described above (Fig. 2), and not an actual need for a specific sequence. We had observed variability when transfections from separate days were compared. We found however that the degree of trans-activation seen on any given day was relatively constant (e.g., Fig. 3). We therefore compared the degree of trans-activation of deleted promoters by doing several measurements of each promoter at the same time (Fig. 3).

In BALB/c 3T3 cells, the sequences necessary for nearmaximal basal expression of the human hsp 70 promoter lie within 67 bases of the start site (Kingston et al. 1986), while a sequence centered at -100 confers heat inducibility (Wu et al. 1986a; see also Fig. 4). Stimulation by the c-myc protein of hsp70 promoters deleted to -84 $(\mathrm{p} \Delta 84)$ and $-120(\mathrm{p} \Delta 120)$ is significantly reduced compared to that seen with a -1250 deletion (Fig. 3). Note that the level of effect of the c-myc protein in these experiments is relatively constant, in contrast to what is observed when transfections from different days are compared (e.g., Fig. 2).

The values in Figure 3 are presented as ratios of the effect of the c-myc protein on expression of the hsp70 promoter as compared with the effect on expression from the MT-I promoter contained in the same transfection. These values therefore include a threefold effect of c-myc on repression of the MT-I promoter. An alternative way to analyze the data is to examine only the effect of the c-myc protein on hsp70-CAT expression. When five experiments are analyzed in this way and averaged, the absolute stimulation of $\mathrm{p} \Delta 1250$ is $5.3, \mathrm{p} \Delta 120$ is stim- 
ulated 2.2-fold, and p $\Delta 84$ is increased 1.5-fold. Sequences between -84 and -120 therefore play a minimal role in stimulation of the human hsp70 promoter by the c-myc protein. These same sequences are primarily responsible for induction of this promoter by heat (Fig. 4; Wu et al. 1986a). These data suggest that the c-myc gene product does not produce its stimulatory effect solely through the heat shock pathway. transcription from the hsp 70 promoter

To characterize the initiation site of hsp 70 transcription in the presence of $\mathrm{c}-\mathrm{myc}$, and to ensure that the level of CAT expression reflects message levels, we performed S1 analysis on total RNA made from BALB/c $3 T 3$ cells 24-48 hr after transfection. The level of appropriately initiated hsp70 transcript increased in the presence of c-myc (Fig. 4, lanes 1 and 2). We used S1 analysis to corroborate that sequences between -84 and -120 played a major role in the heat shock response of this human hsp70 promoter in BALB/c 3T3 cells. The level of appropriately initiated human hsp70 transcription from both $\mathrm{p} \Delta 1250$ and $\mathrm{p} \Delta 120$ was dramatically increased by exposure of transfected cells to $4 \mathrm{hr}$ at $43^{\circ} \mathrm{C}$, while p $\Delta 84$ expression was unaffected (Fig. 4, lanes 9-14).

S1 analysis was next used as a second method of characterizing the sequence requirements for trans-activation of the hsp 70 promoter by the $\mathrm{c}-\mathrm{myc}$ protein. The use of $\mathrm{S} 1$ analysis allowed us to include a pseudo-wildtype promoter in each transfection as an internal reference. This promoter contains sequences to -1250 , but also contains a 33-bp deletion in the $5^{\prime}$ untranslated region of the chimeric hsp70-CAT message (see Fig. 4). When this pseudo-wild-type promoter, contained on plasmid pIR17, is introduced into cells and the resulting RNA is analyzed by S1 analysis, a band of 129 bases results due to looping of the probe sequences opposite the
The c-myc product stimulates appropriately initiated

deletion in the message (Kingston et al. 1986). This band is easily distinguished from the 230-base band representing appropriate initiation from the $\mathrm{p} \Delta$ plasmid. By cotransfecting the pseudo-wild-type promoter with a deleted promoter, we can compare the effect of the c-myc protein on these two promoters in the same cell. If the activation requires sequences that are on the pseudowild-type promoter, but not on the deleted promoter, then expression from the pseudo-wild-type promoter should be preferentially increased by the $\mathrm{c}$-myc protein. This protocol therefore allows us to determine the sequence specificity for activation of the human hsp70 promoter in an internally controlled experiment, eliminating the possibility that the differences in response of a particular deletion to the c-myc are due to transfection variability.

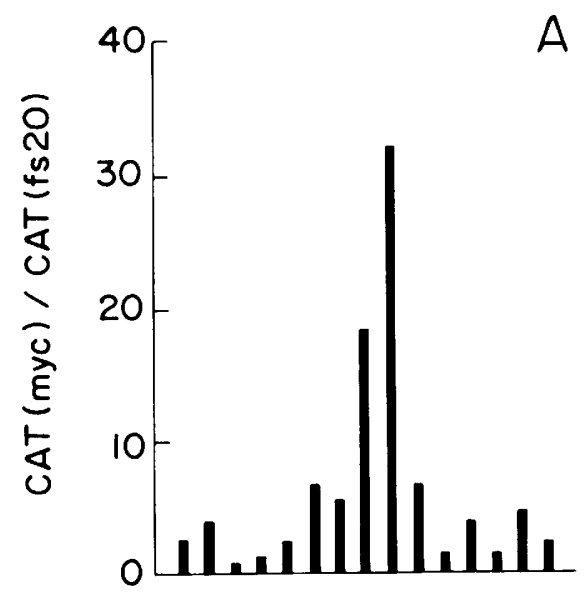

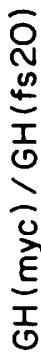

Figure 2. Variability of the regulatory effects of the c-myc protein. Fifteen sets of transfections were carried out on different days in the BALB/c 3T3 cell line. pHSmCAT $(8 \mu \mathrm{g})$ and pXGH5 $(2 \mu \mathrm{g})$ were cotransfected with $5 \mu \mathrm{g}$ of either pSV2cmyc or pfsmyc-20. Forty-eight hours later, the supernatant was assayed for hGH and the cells were lysed and assayed for CAT production. (A) Effect of c-myc on CAT production driven from the hsp 70 promoter. Each bar represents the level of CAT activity seen in the presence of pSV2-cmyc relative to the level seen in the presence of pfsmyc-20 (CATmyc/CATfs). (B) Effect of c-myc on hGH production from the mouse MT-I promoter in the same cells. The bars are a measure of hGH produced in the presence of pSV2-cmyc relative to that produced in the presence of pfsmyc20 (hGHmyc/hGHfs). The ratio of activation of hsp70-CAT to inhibition of MT-I-hGH production, referred to as preferential utilization of hsp70, is shown in Fig. $2 \mathrm{C}$ for the same 15 experiments. The bars are the ratio /CATmyc/ CATfs)/(hGHmyc/hGHfs). All values are the average of duplicate or triplicate experimental dishes. The average value and standard deviation for all of the data in each panel is: $|A| 6.6 \pm 2 ;(B) 0.3 \pm 0.2 ;(C) 17 \pm 10$. The average standard deviation for each data bar (standard deviation between the duplicate or triplicate dishes done on the same day) are: (A) $0.3 ;$ (B) 0.1 ; (C) 0.2 . 


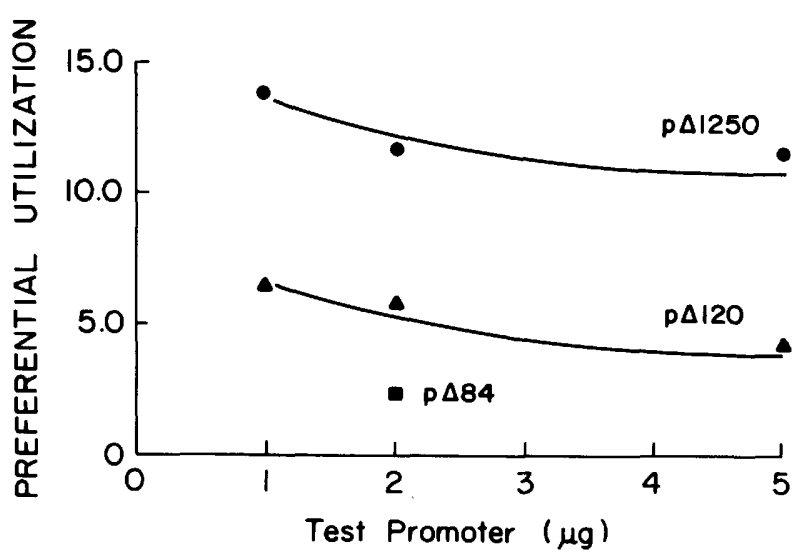

We cotransfected $\mathrm{p} \Delta 120$ and pIR 17 into BALB/c $3 \mathrm{~T} 3$ cells with either pSV2-cmyc or pfsmyc-20. We saw very little effect of the c-myc protein on the level of appropriately initiated hsp 70 message from $\mathrm{p} \Delta 120$ (Fig. 4, lanes 4 and 51, while the level of message from pIR17 in the same transfection was stimulated. Similar results were
Figure 3. Sequence dependence for c-myc stimulation. The hsp70 promoter was deleted from the $5^{\prime}$ side to 120 bp upstream of start site $(\mathrm{p} \Delta 120)$ or to $-84 \mathrm{bp}(\mathrm{p} \Delta 84)$. The specified amounts of the indicated plasmid were transfected wth pXGH5 $(0.5 \mu \mathrm{g})$ and $5 \mu \mathrm{g}$ of either pUC13, pSV2-cmyc, or pfsmyc-20. All transfections were done on the same day into cells split from the same pool. Forty-eight hours after transfection, CAT and hGH levels were determined. The value reported ("preferential utilization") is the ratio of activation of the human hsp70 promoter relative to the mouse MT-I promoter, and is calculated as in the legend to Fig. 2.

obtained with p $\Delta 84$ (Fig. 4, lanes 7 and 8). Note that we observe stimulation of hsp70 message levels by pfsmyc20 , an effect we attribute to vector sequences, as similar effects are seen with pSV2-gpt. These data confirm that sequences upstream of -120 are necessary for full stimulation of the hsp 70 promoter by the $c-m y c$ protein. The

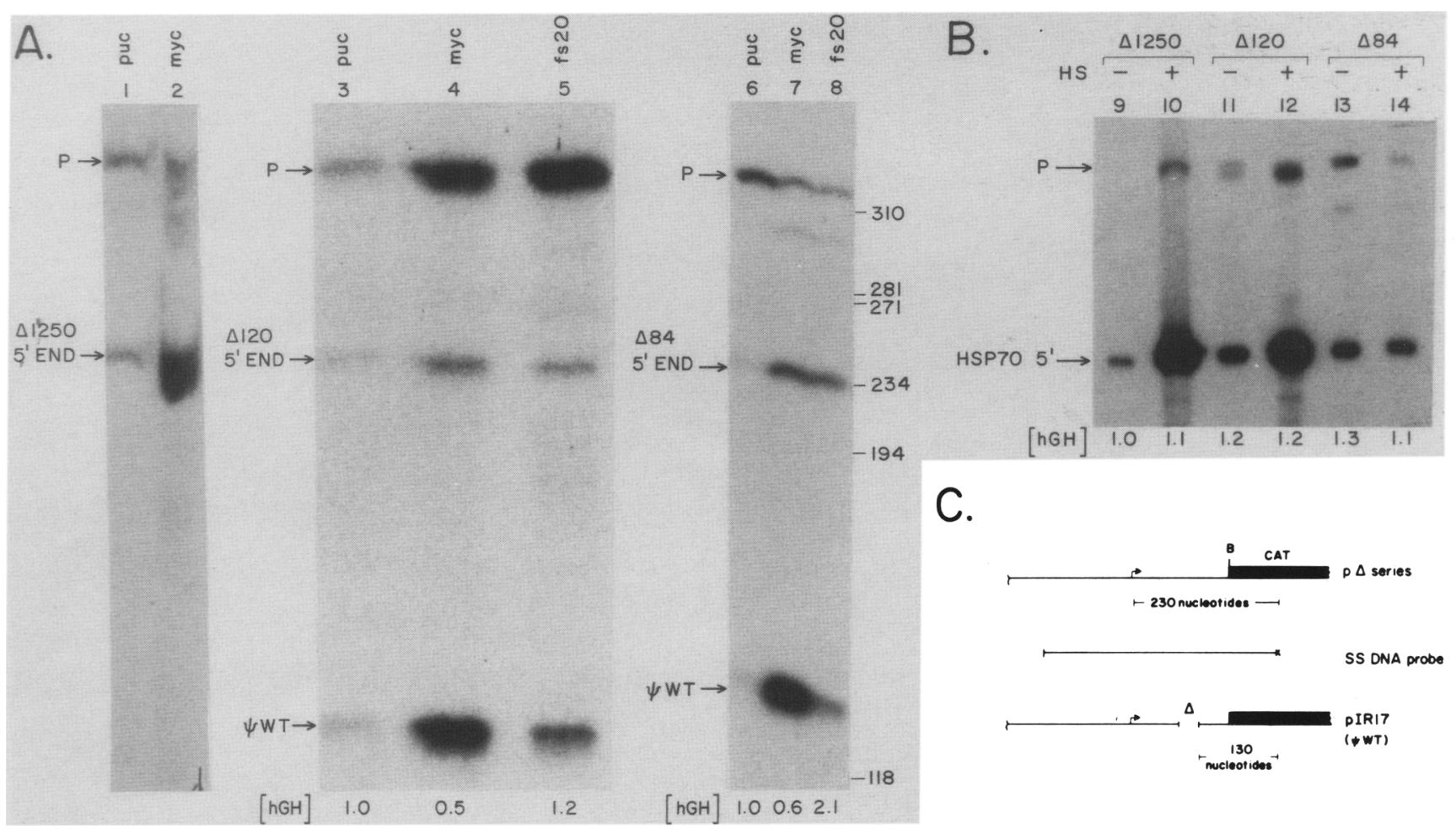

Figure 4. The c-myc protein requires upstream hsp70 promoter sequences to stimulate hsp70 RNA level. (Lanes 1 and 2) BALB/c 3T3 cells were transfected with p $\Delta 1250(8 \mu \mathrm{g})$, and $5 \mu \mathrm{g}$ of either pUCl3 (lane 1) or pSV2-cmyc (lane 2). (Lanes 3-5) Cells were transfected with p $\Delta 120(1 \mu \mathrm{g})$, pIR17 (1 $\mu \mathrm{g})$, pXGH5 (1 $\mu \mathrm{g})$, and $5 \mu \mathrm{g}$ of either pUCl3 (lane 3), pSV2-cmyc (lane 4), or pfsmyc-20 (lane 5). (Lanes 6-8) Cells were transfected with p $\Delta 84(5 \mu \mathrm{g})$, pIR17 $(5 \mu \mathrm{g}), \mathrm{pXGH5}(1 \mu \mathrm{g})$, and $5 \mu \mathrm{g}$ of either pUC13 (lane 6), pSV2-cmyc (lane 7), or pfsmyc-20 (lane 8). (Lanes 9-14) Cells were transfected with pXGH5 $(2 \mu \mathrm{g})$ and $10 \mu \mathrm{g}$ of either p $\Delta 1250$ (lanes 9 and 10$)$, p $\Delta 120$ (lanes 11 and 12), or $\mathrm{p} \Delta 84$ (lanes 13 and 14). Cells in the transfections of lanes 10,12, and 14 were incubated at $43^{\circ} \mathrm{C}$ for $4 \mathrm{hr}$ immediately prior to harvest. Total RNA was harvested 32 (lanes 3-5) or 48 (lanes 1, 2, 6-14) hr after transfection, and was analyzed by S1 analysis using the indicated probe. Plasmid pIR17 contains the complete human hsp70-CAT gene of p $\Delta 1250$, but has a 33-bp deletion in the leader sequence. It thus produces a 130-base protected fragment in the S1 analysis ( $\Psi$ WT). The values at the bottoms of lanes 3-14 are the relative level of hGH expression obtained from plasmid pXGH5 in each transfection. These values are arbitrarily normalized to the level expressed in the experiments of lanes 3 (for lanes 3-5), lane 6 (for 6-9), or lane 9 (for 9-14). 
level of appropriately initiated transcription from $\mathrm{p} \Delta 84$ and $\mathrm{p} \Delta 120$ is similar in the absence of the c-myc protein, verifying that sequences upstream of -84 are not important for basal expression of the promoter in BALB/c 3T3 cells. Expression of hGH from the MT-I promoter decreased in the presence of the c-myc protein in these experiments while expression from the truncated hsp 70 promoters remained constant.

\section{Inhibition of expression from the mouse MT-I promoter}

As described above, the c-myc protein caused on average a three- to fourfold decrease in hGH expression from the mouse MT-I-hGH chimeric gene (Fig. 2B). To confirm that this inhibition reflected the level of appropriately initiated MT-I transcription, S1 nuclease analysis was performed using a probe that extended from +130 to -170 of the MT-I promoter (Fig. 5). We observed good correlation between hGH levels and appropriately initiated MT-I transcripts, implying that hGH expression is an accurate measure of message level of the chimeric genes. These results suggest that the c-myc protein has the ability to inhibit promoter expression. In the experiments described above (Fig. 2), a possible explanation for inhibition of MT-I-hGH expression was that stimulation of the cotransfected human hsp70 promoter by the c-myc protein resulted in a decrease in MT-I expression due to competition for a common transcription factor. We observed, however, a comparable degree of inhibition of the mouse MT-I promoter in the presence of varying concentrations of hsp70 promoter (Fig. 5, lanes $3-7)$, as well as in the absence of any cotransfected hsp70 promoter (Fig. 5, lanes 1 and 2).

Regions of the c-myc gene necessary for trans-activation

The c-myc gene used in the preceding experiments was isolated from a mouse plasmacytoma (Shen-Ong et al. 1982), and produces the characteristic $M_{r} 62,000$ and 64,000 phosphoproteins that have been seen in both human and mouse cells (Wurm et al. 1986). To insure

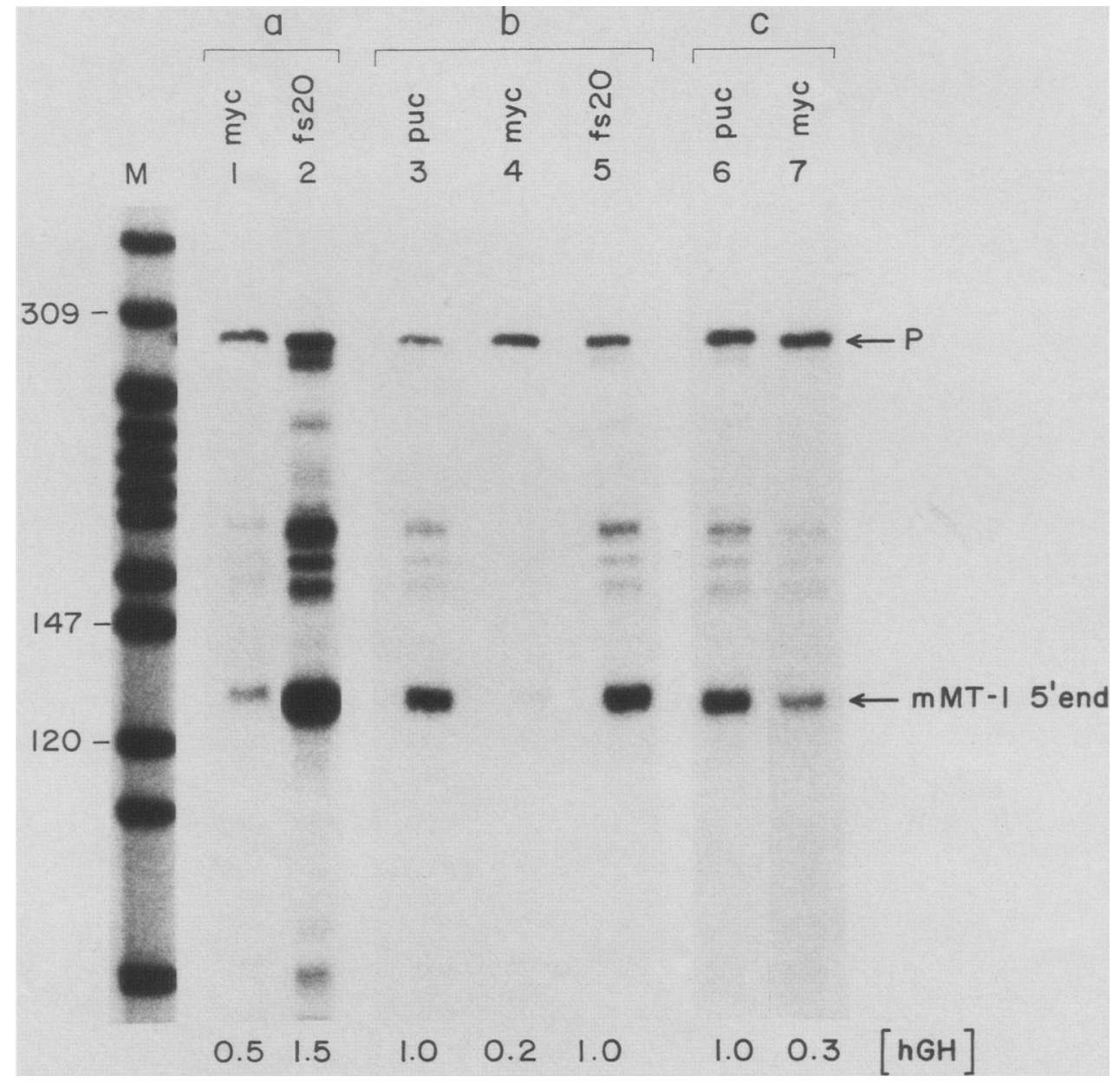

Figure 5. Inhibition of appropriately initiated mouse MT-I transcription by the c-myc protein. Cells were transfected with plasmid pXGH5 $(2 \mu \mathrm{g}$ ) and $5 \mu \mathrm{g}$ of either pUC13 (lanes 3 and 6), pSV2-cmyc (lanes 1, 4, and 7),or pfsmyc-20 (lanes 2 and 5). Included was either no $\mathrm{p} \Delta 1250$ (lanes 1 and 2), $3 \mu \mathrm{g} \mathrm{p} \Delta 1250$ (lanes 3-5), or $8 \mu \mathrm{g} \mathrm{p} \Delta 1250$ (lanes 6 and 7). All transfections except lanes 6 and $7 \mathrm{contain} 10 \mu \mathrm{g}$ total DNA with the remainder made up by pUC13. Total RNA $(30 \mu \mathrm{g})$ was analyzed by S1 analysis using an end-labeled singlestranded probe that extended from +130 (labeled) to -170 of the MT-I-hGH gene. Values at the bottom of each lane are the relative level of hGH expression from plasmid pXGH5, arbitrarily normalized for the level of expression in the experiment of lane 3. 
Table 2. Effects of human and mouse c-myc proteins on gene expression

\begin{tabular}{lrrr}
\hline Effector plasmid & CAT & GH & CAT/GH \\
\hline Set A & & & \\
$\quad$ pSV2-cmyc & $6.4 \pm 0.6$ & $0.5 \pm 0.3$ & $12.8 \pm 4.0$ \\
pfsmyc20 & $1.2 \pm 0.2$ & $1.1 \pm 0.6$ & $1.1 \pm 0.8$ \\
pfsmyc13 & $1.1 \pm 0.2$ & $1.1 \pm 0.5$ & $1.1 \pm 0.7$ \\
Set B & & & \\
pSV7pLHumyc & $5.7 \pm 1.0$ & $0.6 \pm 0.1$ & $9.5 \pm 2.1$ \\
pSV7pLHumyc $\Delta$ pst & $12.0 \pm 0.8$ & $2.0 \pm 0.6$ & $6.0 \pm 1.6$ \\
pSV7pL-mousemyc & $7.4 \pm 1.2$ & $0.3 \pm 0.2$ & $24.6 \pm 3.5$ \\
\hline
\end{tabular}

Transfections contained $\mathrm{p} \Delta 1250(1-2 \mu \mathrm{g})$, pXGH5 $(0.5 \mu \mathrm{g})$, and $5 \mu \mathrm{g}$ of the indicated effector plasmid. The control effector plasmid was either pSV2-gpt (set A) or pSV7pL-polylinker (set B). The values of CAT and GH reported in set A represent two ratios, R1 and R2, respectively, where $\mathrm{R} 1$ = CAT expression in the presence of the indicated effector/CAT expression in the presence of pSV2-gpt plasmid and $\mathrm{R} 2=\mathrm{GH}$ expression in the presence of the indicated effector/GH expression in the presence of pSV2-gpt plasmid. In set B the values are corrected for pSV7pL-polylinker instead of pSV2-gpt. Set A numbers are the average of three experiments done in duplicate and set $\mathrm{B}$ is an average of five experiments. Error values represent the standard deviation over these experiments. Plasmid pfsmyc- 20 and psmyc- 13 contain frameshift mutations at amino acids 40 and 189 , respectively. Plasmid pSV7pL-Humyc $\Delta$ pst contains a deletion of amino acids 40-189. Plasmid pSV7pL-polylinker is the parent plasmid in which the human c-myc gene or its mutants was cloned. Note that all effector molecules are expressed from the SV 40 early promoter.

that the regulatory capabilities reported above reflect a general property of the c-myc protein, we next performed transfections using plasmids that express the human c-myc gene product (Sarid et al. 1986). The human $c-m y c$ protein functioned similarly to the mouse protein: hsp70 expression was stimulated relative to MT-I expression (Table 2). This stimulation was the result of both an increase in hsp70-CAT expression and a decrease in MT-I-hGH expression. We also were interested in determining what regions of the $c-m y c$ protein are necessary for trans-activation. Frameshift mutations at either amino acid 40 (pfsmyc-20) or amino acid 178 (pfsmyc-13) eliminated the ability of the protein product of the mouse gene to regulate expression.

Cotransfection of a construct that contains an internal deletion between amino acids 40 and 178 of the human c-myc gene resulted in activation of the human hsp 70 promoter but no inhibition of the MT-I promoter (Table 2). This deletion leaves exon 3 intact and in frame, but deletes the majority of exon 2 . It is not clear how such an extensive deletion affects the stability of the resultant protein; however, the observation that there is a significant stimulatory effect of this construct suggests that a protein is produced. The observation that this construct has lost the ability to inhibit MT-I expression suggests that the domains responsible for activation and suppression of gene expression by the $c-m y c$ protein are separable.

\section{Regulation of other promoters by the c-myc protein}

The nuclear oncogenes of adenovirus, polyomavirus, and simian virus 40 are capable of regulating gene expression. These gene products all stimulate expression from the adenovirus EII promoter (Jones and Shenk 1979; Berk et al. 1979; Loeken et al. 1986; W. Solomon, pers. comm.). They all inhibit expression from the SV40 early promoter, although by different mechanisms. This inhibition is accomplished by direct binding of the papovavirus $T$ antigens to the promoter, while the EIa proteins inhibit via the SV40 enhancer region (Rio et al. 1980; Borrelli et al. 1984; Velcich and Ziff 1985). In contrast to these viral proteins, the c-myc protein does not stimulate expression from the adenovirus EII promoter (Table 3). We observe instead a slight inhibition. Expression of the $\mathrm{c}-m y c$ protein produces very little change in CAT expression from pSV2-CAT, also in contrast to observations with the viral proteins.

Table 3. The effect of the c-myc protein on expression from the adenovirus EII and SV4O early promoters

\begin{tabular}{lccc} 
& & & CATmyc/CATfs \\
Test promoter & CATmyc/CATfs & GHmyc/GHfs & GHmyc/GHfs \\
\hline p $\Delta$ EII-96-CAT & $0.7 \pm 0.4$ & $0.5 \pm 0.2$ & $1.6 \pm 0.2$ \\
pSV2-CAT & $1.0 \pm 0.6$ & $0.5 \pm 0.2$ & $2.0 \pm 0.8$ \\
\hline
\end{tabular}

Transfections contained $1-8 \mu \mathrm{g}$ of the test promoter (p $\Delta \mathrm{EII}-96-\mathrm{CAT}$ or pSV2-CAT), $1 \mu \mathrm{g}$ of pXGH5, and $5 \mu \mathrm{g}$ of either pSV2-cmyc or pfsmyc-20. The levels of CAT expression and hGH secretion were determined $48 \mathrm{hr}$ after transfection. Error values represent the standard deviation over these experiments. The results are the average of 10 transfection experiments done in duplicate. The expression of CAT from these different promoters is compared with the expression of hGH from the metallothionein promoter in the same transfected cells. The numbers presented are calculated as in the legend to Fig. 2, and reflect the decrease in CAT or GH expression when transfections containing pSV2-cmyc are compared with transfections containing pfsmyc-20. p $\Delta$ EII-96-CAT contains the adenovirus EII promoter fused to CAT, and pSV2-CAT contains the SV40 early promoter fused to CAT. 


\section{Discussion}

Determining the mechanism by which oncogenes exert their phenotype is of fundamental importance to understanding neoplastic transformation. The c-myc product has been found to reside in the nucleus and to bind DNA. These observations, coupled with the observation that the c-myc protein can stimulate expression from a Drosophila hsp70 promoter region, have raised the hypothesis that the c-myc protein functions as a regulator of mammalian transcription.

The c-myc protein has a novel trans-activation function

We demonstrate here that expression of the c-myc protein in mammalian cells increases appropriately initiated transcription from a human hsp70 promoter. This activation requires sequences upstream of the initiation site, suggesting that stimulation is a result of increased transcription, not increased stability, transport, or translation of the message. How does this activation occur? The mechanism by which other nuclear oncogenes stimulate expression from cellular promoters appears at this point to be indirect, as sequences required for stimulated expression have not been separated from those required for basal expression (Green et al. 1983; Brady et al. 1985; Keller and Alwine 1985; Kingston et al. 1986; Kovesdi et al. 1986; SivaRaman et al. 1986; for review, see Kingston et al. 1985).

The c-myc protein modulates gene expression by a mechanism different than that of previously characterized viral trans-activating proteins, however. The sequences required for stimulation of hsp70 expression by the c-myc protein are different from those required for stimulation of this same promoter by the early region proteins of polyomavirus and simian virus 40 (Kingston et al. 1986; W. Solomon, unpubl.). These latter proteins do not need sequences upstream of -67 for activation, while the c-myc protein requires sequences upstream of -84 , and hence upstream of the elements required for basal expression of this promoter in mouse cells (Fig. 4). In addition, the c-myc protein slightly represses expression from the adenovirus EII promoter and has very little effect on the SV40 early promoter. These latter effects contrast with the effects of the adenovirus EIa proteins and the papovavirus $T$ antigens of these promoters. Therefore, one cannot infer the mechanism of trans-activation by the c-myc protein from present studies on these other trans-activating proteins.

Does the c-myc protein interact directly with the hsp70 promoter to activate it, or does it indirectly activate through other factors? One possibility is that production of the c-myc protein in our experiments stresses the cell, and thus activates the hsp70 promoter via the heat shock response. The regulatory element responsible for heat induction of promoters is well characterized, and a perfect fit to the consensus sequence is found centered at -100 of the promoter used in this work (Mirault et al. 1982; Pelham 1982, 1985; Hunt and Morimoto 1985). This single element is primarily responsible for heat induction of this promoter, as evidenced by the dramatic difference in heat inducibility between $\mathrm{p} \Delta 84$ and p $\Delta 120$ (Fig. 4; see also $W u$ et al. 1986a). Sequences between -84 and -120 play very little role in induction of the human hsp70 promoter by c-myc (Figs. 3 and 4), while sequences upstream of -120 play a major role in c-myc induction. Because there is a minimal effect of sequences upstream of -120 on the magnitude of heat shock induction of this promoter (Fig. 4; J. Greene, unpubl.), it is impossible rigorously to exclude some role for the elements of the heat shock response in stimulation of this human hsp70 promoter by c-myc. The data, however, argue that the c-myc protein stimulates hsp70 transcription by a separate mechanism

The sequences required for c-myc stimulation are also distinct from those required for serum induction of the promoter (Wu et al. 1986a). Activation of this promoter by the c-myc protein thus has different sequence requirements than induction by any previously characterized effector. The data therefore suggest that the c-myc protein either interacts directly with the promoter or activates via a novel pathway involving other factors. The assays for trans-activation by the c-myc protein described here in combination with biochemical studies involving purified $\mathrm{c}-\mathrm{myc}$ protein should allow dissection of the mechanism by which this cellular oncogene stimulates gene expression.

\section{Inhibition of expression from the mouse MT-I promoter region}

Expression of the c-myc protein inhibits the level of appropriately initiated transcripts from the mouse MT-I promoter. Two prominent explanations exist for this phenomena; either the c-myc protein specifically represses expression from this promoter region or the inhibition is an indirect result of a cytotoxic effect of c-myc in transfected cells. Overproduction of the c-myc protein can result in cell death under certain conditions (Wurm et al. 1986). Determination of whether the level of c-myc expression achieved in the transfection experiments described here results in cell death is problematic due to the low percentage of cells that express transfected DNA. However, when we perform cotransfection experiments with a mutant hsp70 promoter $(\mathrm{p} \Delta 84)$ containing only sequences necessary for basal expression, hsp70 expression remains constant while MT-I-hGH expression decreases (Fig. 4). Furthermore, both the SV40 early and adenovirus EII promoters are minimally affected by the c-myc protein in experiments where we observe inhibition of the MT-I promoter (Table 3). These results argue that specific components in the MT-I promoter are required for inhibition, and that the effect is not one of general cytotoxicity. An alternative interpretation, that inhibition of the MT-I promoter is caused by cytotoxicity while the differential effects of the human hsp70, EII, and SV40 early promoters are due to a specific ability to stimulate these three promoters, would appear less likely.

\section{Modulation of gene expression and transformation}

The implication that the c-myc protein can both positively and negatively regulate gene expression raises the 
hypothesis that neoplastic transformation is a direct effect of these regulatory capabilities. In this study, we report that a c-myc gene deleted for most of exon 2 is capable of stimulating hsp70 expression, but appears incapable of inhibiting MT-I expression (Table 2). This mutant is not capable of collaborating with the EJ-ras oncogene to transform rat embryo fibroblasts (Sarid et al. 1987). These data may be taken to suggest that the ability to inhibit expression from the MT-I promoter is more relevant to transformation than the ability to stimulate hsp70 expression. One must be cautious, however due to the inherent differences in sensitivity of the transformation and regulatory assays. A more detailed analysis of mutations in c-myc that affect these functions will be necessary to correlate these two regulatory activities with an ability to transform cells.

The adenovirus EIa region shares limited homology with the c-myc gene (Ralston and Bishop 1983), behaves similarly in the ability to induce various transformed phenotypes (Ruley 1983; Land et al. 1983), and shares an ability to modulate gene expression positively and negatively. The regions of the adenovirus EIa proteins necessary for trans-activation do not correlate well with those necessary for collaboration with EJ-ras in transformation of rat embryo fibroblasts. Interestingly, there is a better correlation between transformation potential and the ability to inhibit expression from the SV40 early promoter (Lillie et al. 1986). As detailed above, the mechanism by which the c-myc protein regulates gene expression appears to differ from that by which the adenovirus EIa proteins regulate gene expression. This latter consideration suggests that there is not a common regulatory capability shared by these nuclear oncogenes that is responsible for the transformation phenotype of these proteins. The observation that many nuclear-localized oncogenes are able to modulate gene expression, however, argues that this ability is important to the function of these proteins. Whether the regulatory abilities of the c-myc protein play a role in cellular transformation awaits further study.

\section{Methods \\ Cells and transfections}

BALB/c 3T3 cells were cultured in Dulbecco's modified Eagle's medium (DMEM) supplemented with $10 \%$ calf serum in $10-\mathrm{cm}$ plates. Cells were used for no longer than 10 passages, and were carefully passaged to avoid confluence. Transfections were carried out using a modification of the calcium phosphate precipitation procedure of Graham and van der $\mathrm{Eb}$ (1973; Kingston et al. 1986). Total transfected DNA $(8-15 \mu \mathrm{g})$ was ethanol-precipitated and resuspended in $0.5 \mathrm{ml}$ of $250 \mathrm{mM} \mathrm{CaCl}_{2}$. The DNA solution was added dropwise to $0.5 \mathrm{ml}$ of buffered $2 \times$ HEPESbuffered saline (HeBS). The precipitate was immediately vortexed for $5 \mathrm{sec}$ and allowed to sit at room temperature for 20 min. This solution was then added to a $10-\mathrm{cm}$ dish of cells (20-40\% confluent) that had been fed with fresh media $2 \mathrm{hr}$ earlier. Sixteen hours later, the cells were washed with phosphate-buffered saline (PBS) and fed with fresh media. Cells were harvested $8-32 \mathrm{hr}$ after removing the precipitate for either protein or RNA studies. Cells were not allowed to reach con- fluence before harvest. Transfections were done in duplicate or triplicate.

\section{Plasmids}

Construction of all plasmids used is described in detail elsewhere. Plasmids contain the following sequences.

$\mathrm{p} \Delta$ series (Kingston et al. 1986): The human hsp70 promoter (Wu et al. 1985) is fused to the structural gene of pSV2-CAT (Gorman et al. 1982) at a BamHI site at +160 of the human 5' untranslated region. Plasmids contain the indicated number of bases $5^{\prime}$ of the transcription initiation site $(\mathrm{p} \Delta 84$ contains sequences to -84 ).

pXGH5 (Selden et al. 1986): The mouse MT-I promoter is fused to the coding region of the hGH gene.

pSV2-cmyc (Land et al. 1983): Exons 2 and 3 of a mouse c-myc gene isolated from a plasmacytoma (Shen-Ong et al. 1982) are fused to the SV40 early promoter.

pfsmyc-20 and pfsmyc-13 (Kingston et al. 1984a): Both are derived from pSV2-cmyc by deleting the four internal bases of either the promoter proximal (pfsmyc-20) or distal (pfsmyc-13) PstI site in exon 2.

pSV7PL myc series (Sarid et al. 1986): Exons 2 and 3 of either the mouse c-myc gene (mousemyc) or the human c-myc (Humyc) are fused to the SV40 early promoter region. Plasmid pSV7PLHumyc $\Delta$ pst contains a deletion of the sequences between the two PstI sites in exon 2. This deletion leaves the carboxy-terminal portion of the protein in frame.

p $\Delta$ EII-96-CAT (Kingston et al. 1984b): The adenovirus EII promoter, containing 96 bases of $5^{\prime}$-flanking sequence, is fused to the CAT gene.

pSV2-CAT (Gorman et al. 1982): The SV40 early promoter is fused to the CAT gene.

All plasmid DNA was grown using M9 minimal media and purified by banding twice by ethidium bromide- $\mathrm{CsCl}$ gradient centrifugation. DNA concentrations were determined spectrophotometrically and were verified by restriction digest and agarose gel electrophoresis.

\section{RNA preparation and analysis}

Total cellular RNA was prepared by the guanidium $/ \mathrm{CsCl}$ method described elsewhere (Ullrich et al. 1977; Maniatis et al. 1982). Single-stranded end-labeled probes were prepared as described previously (Kingston et al. 1986). The human hsp70 probe contains bases +229 (labeled) to -133 of the hsp70CAT fusion gene.

S1 nuclease analysis (Berk and Sharp 1977) was performed as follows: $10-30 \mu \mathrm{g}$ total cellular RNA was mixed with $3 \times 10^{4}$ $\mathrm{dpm}$ probe, ethanol-precipitated, and resuspended in hybridization buffer (40 mM Pipes (pH 6.4), $1 \mathrm{~mm}$ EDTA, $0.4 \mathrm{M} \mathrm{NaCl}$, $80 \%$ formamide). Hybridization was $30^{\circ} \mathrm{C}$ for $16 \mathrm{hr}$. S1 nuclease digestion and analysis on $8 \%$ denaturing polyacrylamide gels was by standard procedures (Maniatis et al. 1982).

\section{Determination of $G H$ expression levels}

At the time of harvest of transfected cells, hGH levels in the media were measured using a solid-phase two-site radioimmunoassay kit under the conditions recommended by the manufacturer (Hybritech Inc.). Standards were used to verify that all values were within the linear range of the assay. 


\section{Determination of CAT activity in transfected cells}

At the time of harvesting, cells were washed thoroughly with ice-cold PBS, lysed by three rounds of freeze-thaw in $250 \mathrm{~mm}$ Tris buffer, and the supernatant was analyzed for CAT activity using the standard procedure (Gorman et al. 1982). Serial dilutions were performed to bring the value in the assay to under $30 \%$ conversion to the acetylated form. Activities reported are corrected for any dilution required to bring the assay into this range.

\section{Acknowledgments}

We are grateful to P. Sharp, in whose laboratory this work was initiated, for his continued support and advice. We thank J. Sarid and R. Selden for donation of plasmids, D. Moore for fruitful discussions, R. Brent for critical comments on the manuscript, and R. Hyde for preparing the manuscript. This work was supported by a grant from Hoechst AG.

\section{References}

Abrams, H.D., L.R. Rohrschneider, and R.N. Eisenman. 1982. Nuclear location of the putative transforming protein of avian myelocytomatosis virus. Cell 29: 427-439.

Alitalo, K., G. Ramsay, J.M. Bishop, S.O. Pfeifer, W.W. Colby, and A.D. Levinson. 1983. Identification of nuclear proteins encoded by viral and cellular myc oncogenes. Nature 306: 274-277.

Berk, A.J. and P.A. Sharp. 1977. Sizing and mapping of early adenovirus mRNAs by gel electrophoresis of Sl endonuclease digested hybrids. Cell 12: 721-731.

Berk, A.J., F. Lee, T. Harrison, J. Williams, and P.A. Sharp. 1979. Pre-early adenovirus $5^{\prime}$ gene product regulates synthesis of early viral messenger RNAs. Cell 17: 935-944.

Borrelli, E., R. Hen, and P. Chambon. 1984. Adenovirus-2ElA products repress enhancer-induced stimulation of transcription. Nature 312: 608-612.

Brady, J., M.R. Loeken, and G. Khoury. 1985. Interaction between two transcriptional control sequences required for tumor-antigen mediated simian virus 40 late gene expression. Proc. Natl. Acad. Sci. 82: 7299-7303.

Denoto, F.M., D.D. Moore, and H.M. Goodman. 1981. Human growth hormone DNA sequence and mRNA structure: Possible alternative splicing. Nucleic Acids Res. 9: 3719-3730.

Donner, P., I. Greiser-Wilke, and K. Moelling. 1982. Nuclear localization and DNA binding of the transforming gene product of avian myelocytomatosis virus. Nature 296: 262266.

Durnam, D.M., F. Perrin, F. Gannon, and R. Palmiter. 1980. Isolation and characterization of the mouse metallothionein I gene. Proc. Natl. Acad. Sci. 77: 6511-6515.

Gorman, C.M., L.F. Moffat, and B.H. Howard. 1982. Recombinant genomes which express chloramphenicol acetyl transferase in mammalian cells. Mol. Cell. Biol. 2: 1044-1051.

Graham, F.L. and A.J. van der Eb. 1973. A new technique for the assay of infectivity of human adenovinus 5 DNA. Virology 52: 456-467.

Green, M.R., R. Treisman, and T. Maniatis. 1983. Transcriptional activation of cloned human $\beta$-globin genes by viral immediate-early gene products. Cell 35: 137-148.

Hamer, D.H. and M. Walling. 1982. Regulation in vivo of a mammalian gene: Cadmium induction of a mouse metallothionein gene in SV40 vectors. I. Mol. Appl. Genet. 1: $273-288$.

Hann, S.R., H.D. Abrams, L.R. Rohrschneider, and R.N.
Eisenman. 1983. Proteins encoded by v-myc and c-myc oncogenes: Identification and localization in acute leukemia virus tranformants and bursal lymphoma cell lines. Cell 34: 789-798.

Hunt, C. and R.I. Morimoto. 1985. Conserved features of the nucleotide sequence of human hsp70. Proc. Natl. Acad. Sci. 82: 6455-6459.

Imperiale, M.J., H.T. Kao, L.T. Feldman, J.R. Nevins, and S. Strickland. 1984. Common control of the heat shock gene and early adenovirus genes: Evidence for a cellular EIa-like activity. Mol. Cell. Biol. 4: 867-874.

Jones, N. and T. Shenk. 1979. An adenovirus 5 early gene function regulates expression of other early viral genes. Proc. Natl. Acad. Sci. 76: 3665-3669.

Kao, N.T. and J.R. Nevins. 1983. Transcriptional activation and subsequent control of the human heat shock gene during adenovirus infection. Mol. Cell. Biol. 3: 2058-2065.

Keller, J.M. and J.C. Alwine. 1985. Analysis of an activatable promoter: Sequences in the simian virus 40 late promoter required for $\mathrm{T}$ antigen mediated transactivation. Mol. Cell. Biol. 5: 1859-1869.

Khandjian, E.W. and H. Turler. 1983. Simian virus 40 and polyomavirus induce synthesis of heat shock proteins in mammalian cells. Mol. Cell. Biol. 3: 1-8.

Kingston, R.E., A.S. Baldwin, Jr., and P. Sharp. 1984a. Regulation of heat shock protein 70 gene expression by c-myc. $\mathrm{Na}$ ture 312: 280-282.

Kingston, R.E., R.J. Kaufman, and P.A. Sharp. 1984b. Regulation of transcription of the adenovirus EII promoter by EIa gene products: Absence of sequence specificity. Mol. Cell. Biol. 4: 1970-1977.

Kingston, R.E., A.S. Baldwin, and P.A. Sharp. 1985. Transcription control by oncogenes. Cell 41: $3-5$.

Kingston, R.E., A. Cowie, R.I. Morimoto, and K.A. Gwinn. 1986. Binding of polyomavirus large $T$ antigen to the human hsp70 promoter is not required for trans-activation. Mol. Cell. Biol. 6: 3180-3190.

Kovesdi, I., R. Reichel, and J.R. Nevins. 1986. EIa transcription induction: Enhanced binding of a factor to upstream promoter sequences. Science 231: 719-722.

Land, H., L.F. Parada, and R.A. Weinberg. 1983. Tumorigenic conversion of primary embryo fibroblasts requires at least two cooperating oncogenes. Nature 304: 596-601.

Lillie, J.W., M. Green, and M.R. Green. 1986. An adenovirus EIa protein region required for transformation and transcriptional repression. Cell 46: 1043-1051.

Loeken, M.R., G. Khoury, and J. Brady. 1986. Stimulation of the adenovirus E2 promoter by simian virus $40 \mathrm{~T}$ antigen or EIa occurs by different mechanisms. Mol. Cell. Biol. 6: 20202026.

Maniatis, T., E. Fritsch, and J. Sambrook. 1982. Molecular cloning: A laboratory manual. Cold Spring Harbor Laboratory, Cold Spring Harbor, New York.

Mirault, M.-E., R. Southgate, and E. Delwart. 1982. Regulation of heat shock genes: A DNA sequence upstream of Drosophila hsp70 genes is essential for their induction in monkey cells. EMBO I. 1: 1279-1285.

Nevins, J.R. 1982. Induction of the synthesis of a 70,000 dalton mammalian heat shock protein by the adenovirus EIa gene product. Cell 29: 913-919.

Pelham, H.R.B. 1982. A regulatory upstream promoter element in the Drosophila hsp70 gene. Cell 30: $517-528$.

. 1985. Activation of heat-shock genes in eukaryotes. Trends Genet. 1: 31-35.

. 1986. Speculations on the functions of the major heat shock and glucose regulated proteins. Cell 46: 959-961. 
Persson, H. and P. Leder. 1984. Nuclear localization and DNA binding properties of a protein expressed by human c-myc oncogene. Science 225: 718-721.

Ralston, R. and J.M. Bishop. 1983. The protein products of the $m y c$ and myb oncogenes and adenovirus EIa are structurally related. Nature 306: $803-806$.

Rio, D., A. Robbins, R. Myers, and R. Tjian. 1980. Regulation of simian virus 40 early transcription in vitro by a purified tumor antigen. Proc. Natl. Acad. Sci. 77: 5706-5710.

Ruley, E.H. 1983. Adenovirus early region $1 \mathrm{~A}$ enables viral and cellular transforming genes to transform primary cells in culture. Nature 304: 602-606.

Sarid, J., T. Halazonetis, W. Murphy, and P. Leder. 1987. Evolutionarily conserved regions of the human c-myc protein can be uncoupled from transforming activity. Proc. Natl. Acad. Sci. 84: 170-173.

Selden, R.F., K. Burke-Howie, M.E. Rowe, H.M. Goodman, and D.D. Moore. 1986. Human growth hormone as a receptor gene in regulation studies using transient growth expression. Mol. Cell. Biol. 6: 3173-3179.

Shen-Ong, G.L.C., E.J. Keath, S.P. Piccoli, and M.D. Cole. 1982. Novel myc oncogene RNA from abortive immunoglobulingene recombination in mouse plasmacytomas. Cell 31: $443-450$.

SivaRaman, L., S. Subramanian, and B. Thimmappaya. 1986. Identification of a factor in HeLa cells specific for an upstream transcriptional control response of an Ela-inducible adenovirus promoter and its relative abundance in infected and uninfected cells. Proc. Natl. Acad. Sci. 83: 5914-5918.

Ullrich, A., J. Shine, J. Shirgwin, R. Pictet, E. Tischer, W.I. Rutter, and H.M. Goodman. 1977. Rat insulin genes: Constructions of plasmids containing the coding sequences. Science 196: 1313-1318.

Velcich, A. and E. Ziff. 1985. Adenovirus Ela proteins repress transcription from the SV40 early promoter. Cell 40: 705716.

Wu, B.J. and R.I. Morimoto. 1985. Transcription of the human hsp70 gene is induced by serum stimulation. Proc. Natl. Acad. Sci. 82: 6070-6074.

Wu, B., C. Hunt, and R.I. Morimoto. 1985. Structure and expression of the human gene encoding major heat shock protein hsp70. Mol. Cell. Biol. 5: 330-341.

Wu, B.J., R.E. Kingston, and R.I. Morimoto. 1986a. The human hsp70 promoter contains at least two distinct regulatory domains. Proc. Natl. Acad. Sci. 83: 629-633.

Wu, B.J., H.C. Hurst, N.C. Jones, and R. Morimoto. 1986b. The EIA $13 S$ product of adenovirus 5 activates transcription of the cellular human hsp70 gene. Mol. Cell. Biol. 6: 29942999.

Wurm, F.W., K.A. Gwinn, and R.E. Kingston. 1986. Inducible overproduction of the mouse c-myc protein in mammalian cells. Proc. Natl. Acad. Sci. 83: 5414-5418. 


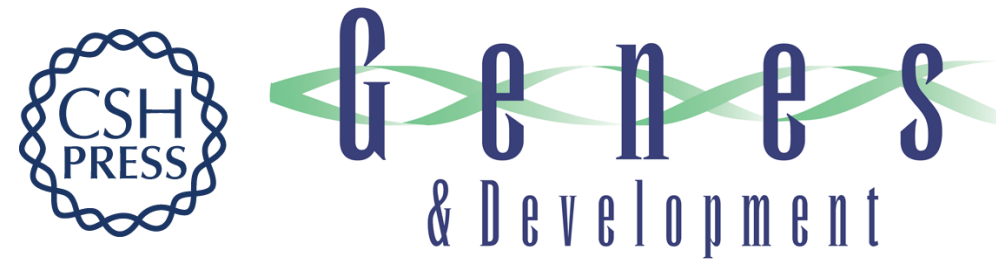

\section{Activation and repression of mammalian gene expression by the c-myc protein.}

R Kaddurah-Daouk, J M Greene, A S Baldwin, et al.

Genes Dev. 1987, 1:

Access the most recent version at doi:10.1101/gad.1.4.347

References This article cites 48 articles, 24 of which can be accessed free at:

http://genesdev.cshlp.org/content/1/4/347.full.html\#ref-list-1

License

Email Alerting

Service

Receive free email alerts when new articles cite this article - sign up in the box at the top right corner of the article or click here.

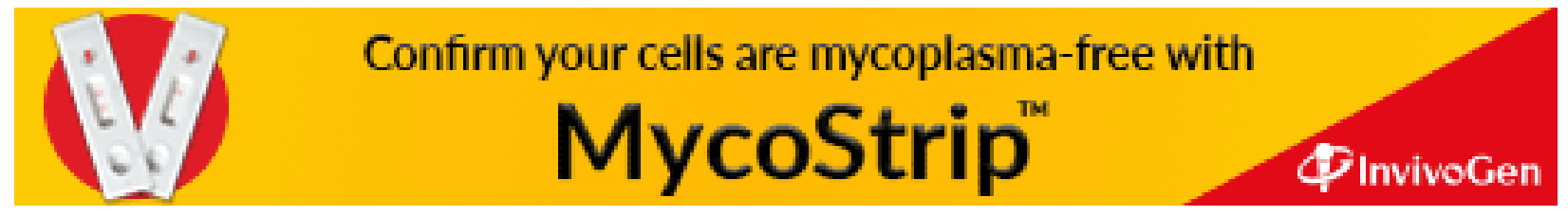

